\title{
FORCED MOTION CFD SIMULATION AND LOAD REFINEMENT EVALUATION OF FLOATING VERTICAL-AXIS TIDAL CURRENT TURBINES
}

\author{
Wanchao Zhang \\ Jiangsu University of Science and Technology, China \\ Yujie Zhou \\ Jiangsu University of Science and Technology, China \\ Kai Wang \\ Wuhan Second Ship Design \& Research Institute, China \\ Xiaoguo Zhou \\ Jiangsu University of Science and Technology, China
}

\begin{abstract}
Simulation of the hydrodynamic performance of a floating current turbine in a combined wave and flow environment is important. In this paper, ANSYS-CFX software is used to analyse the hydrodynamic performance of a vertical-axis turbine with various influence factors such as tip speed ratio, pitching frequency and amplitude. Time-varying curves for thrust and lateral forces are fitted with the least squares method; the added mass and damping coefficients are refined to analyse the influence of the former factors. The simulation results demonstrate that, compared with nonpitching and rotating turbines under constant inflow, the time-varying load of rotating turbines with pitching exhibits an additional fluctuation. The pitching motion of the turbine has a positive effect on the power output. The fluctuation amplitudes of thrust and lateral force envelope curves have a positive correlation with the frequency and amplitude of the pitching motion and tip speed ratio, which is harmful to the turbine's structural strength. The mean values of the forces are slightly affected by pitching frequencies and amplitudes, but positively proportional to the tip speed ratio of the turbine. Based upon the least squares method, the thrust and lateral force coefficients can be divided into three components, uniform load coefficient, added mass and damping coefficients, the middle one being significantly smaller than the other two. Damping force plays a more important role in the fluctuation of loads induced by pitching motion. These results can facilitate study of the motion response offloating vertical-axis tidal current turbine systems in waves.
\end{abstract}

Keywords: vertical-axis tidal current turbine, hydrodynamic loads, pitching motion, added mass, damping coefficients

\section{INTRODUCTION}

Traditional fossil fuels account for a major part of the world's energy consumption, but we will no longer use fossil fuels in the next few years, according to the current mining rates [17]. Therefore, more and more countries in the world have begun to develop clean and renewable energy sources, and as a kind of marine renewable energy, tidal current energy is gradually entering people's vision.

As the main development device of tidal current energy, tidal current turbines can be divided into vertical- and horizontal-axis turbines according to the relative location of the main shaft and flow direction [2]. Compared to a horizontal-axis turbine, a vertical-axis turbine has the following characteristics: 1 . the rotating direction is not affected by flow direction, and there is no yaw mechanism; 2. easy processing and low cost because of simple blade structure; 3 . the power generation system and speedincreasing mechanism can be installed above the surface to reduce the difficulty of underwater sealing.

At present, the main theories used to study the hydrodynamics of vertical-axis turbines are flow tube theory, vortex theory, experimental and CFD (computational fluid dynamics) methods. Compared with the former two theories, the ability of CFD to capture more instantaneous flow field information is the biggest advantage. Experiments are not massively used because of their high expenditure. Furthermore, a full-scale model can be simulated by 
using CFD. Taking these advantages into consideration, currently CFD is extensively employed in research concerning vertical-axis tidal current turbines $[13,17]$.

Over the past several decades, vertical-axis tidal current turbines rotating with a fixed upright axis have been the main research subjects, and a mass of research achievements has been captured. Li and Calisal $[8,9]$ examined the influence of the incoming flow angle, relative distance and rotating direction on the power output and torque fluctuation by modelling twin-turbine systems in 2009-2010. The results showed that the total power output of a twin-turbine system with optimal layout can be about $25 \%$ higher than two times that of a stand-alone turbine, and when the co-rotating system is optimally configured, the downstream turbine should be partially in the wake of the upstream turbine. In 2011, Li [10] conducted a series of experiments with vertical-axis turbines to research the hydrodynamic influence of density, leaf number, pitch angle and chord length, and then compared the results with those from a numerical simulation to validate the reliability of CFD. It showed that the SST turbulence model can provide higher computational accuracy, and the grid has little influence when $\mathrm{Y}^{+} \leq 2$. His research also proved that fixed pitch angle turbines are poor at self-starting, but free variable pitch angle turbines can self-start quickly and have higher energy utilization. In 2014, Li et al. [4] developed a hybrid approach that combines a discrete vortex method with a finite element method that can simulate the integrated hydrodynamic and structural response of a vertical-axis turbine. They discovered that the optimization of a threeblade vertical-axis turbine design using the hybrid method yielded a turbine $\mathrm{H} / \mathrm{R}$ ratio of about 3.0 for reliable maximum power output.

In summary, these researches mentioned above are on vertical-axis turbines revolving around a fixed upright axis. In fact, the floating platform of the floating tidal current power station will undergo six degrees of freedom motion induced by waves, and the axis of turbines will be skewed to the incoming flow. Recently, little research on wave-induced motions about vertical-axis tidal current turbines has been done. However, horizontal-axis tidal turbines can offer us a series of predeterminations and experiences. In 2010, Galloway et al. [3] conducted experimental research on three-blade horizontal-axis tidal turbines in regular waves and deep water (wave height $=0.08 \mathrm{~m}$, inflow velocity $=1.5 \mathrm{~m} / \mathrm{s}$ ); the results showed that the mean parameters (thrust and torque) are the same with or without waves, but the transient values were highly influenced by waves: the fluctuation of thrust increased by $37 \%$ and torque reached up to $35 \%$. The analogy analysis method can also be used to predict the change regulation of vertical-axis turbine parameters in waves. In fact, vertical-axis turbines will move with the platform, and then the velocity distribution around the turbine also alters with different distributions of wave height and frequency. Therefore, as with a horizontal-axis tidal turbine, the load and torque of the turbine must also fluctuate greatly.

Study of vertical-axis wind turbine rotated in oblique flow or tilted conditions can provide some creative proposals for the study of vertical-axis tidal current turbines. In 2016, Chowdhury et al. [1] carried out a numerical validation of an existing experimental work on vertical axis wind turbines (VAWT) in upright and tilted conditions. The numerical validation was accomplished by means of CFD analysis; mesh dependency analysis and optimum time step were also conducted. The results showed that the result obtained from SST k-omega was closest to the experimental one and a VAWT in the tilted condition produces greater torque downwind in comparison to an upright one. The wake of a tilted-axis turbine would proceed downstream in a tilted manner. As a result, the wake of a tilted turbine would be shifted downward. In 2011, the vorticity transport model was used to simulate aerodynamic performance and wake dynamics by Scheurich and Brown [14]. The results partly confirmed previous experimental measurements and suggested that a straight-bladed vertical-axis wind turbine operated in oblique flow might produce a higher power coefficient than when it is operated in normal flow. This is because oblique flow skews the convection of the wake, which allows a significant portion of the blade to operate. In such a case, over its entire azimuth in a flow region, the influence of the wake is significantly reduced compared to the situation in normal flow. VAWT and tidal current turbines have almost the same operating principles. Therefore, studying VAWT in tilted flow conditions can provide constructive comments on the research on tidal current turbines rotating around a skewed axis. Later in 2017, Zhang et al. [20] established a 3-D calculation model in the CFD software and evaluated the effect of extension length on the hydrodynamic loads and the wake field. In 2018, Li et al. [6] further explored a verticalaxis tidal turbine array with a single blade and studied the phenomenon of hydrodynamic interference of the tidal turbine array based upon their former proposed unsteady boundary element model [5]. Later they further explored the effect of the configuration parameters on the verticalaxis tidal turbine and obtained the hydrodynamic loads characteristics [7]. Tomporowski [16] designed a floating and innovative water turbine to convert the mechanical energy to electrical energy and analyzed the effect of geometrical and dynamic features on the pressures, forces and torques.

However, most of the current research on turbines focuses on the conversion efficiency of the turbine under environmental conditions such as different velocity or deflection angle, and there are few reports on load refinement evaluation of turbines in additional wave environments. Especially when the coupling model of the turbine and the floating platform is placed in a wave and current environment, research on its complex motion and hydrodynamic characteristics is worthy of attention. In fact, a tidal power station in practical application is often accompanied by the action of waves. Therefore, it is very important for the design, construction and application of tidal power stations to simulate the motion of a tidal power station in a complex marine environment based on current software functions. This paper studied the hydrodynamic performance of a vertical-axis turbine with compulsorily 
pitching motion under the steady inflow condition using ANSYS-CFX. The axis of a vertical-axis tidal current turbine is assumed to stay in the tilted condition during pitching motion in waves. A series of simulated working conditions with several tip speed ratios, frequencies and amplitudes was conducted. The time-varying hydrodynamics curves for thrust and lateral forces were measured, and fitted to get the added mass and damping coefficients by using the least squares fitting method. This research can provide vital data for research on wave-induced responses of floating support structures for tidal current turbines.

\section{NUMERICAL SIMULATIONS}

\section{BASIC THEORIES}

The incoming flow velocity of the turbines is represented as $V$, the diameter of the turbine as $D$, the number of blades as $Z$ and the rational speed of the turbine as $\omega$; the positive direction of the $\mathrm{X}$-axis is the inflow direction. The relative parameters which will be employed in the following text are given in Table 1. $C_{F X}, C_{F Y}$ and $C_{P}$ are essential indices for assessing the hydrodynamic performance of a turbine.

Tab. 1. Nomenclature for turbines

\begin{tabular}{|c|l|c|l|}
\hline Symbol & \multicolumn{1}{|c|}{ Explanation } & Symbol & \multicolumn{1}{|c|}{ Explanation } \\
\hline$V$ & Incoming flowing velocity & $F_{X}$ & Thrust of turbine \\
\hline$D$ & Diameter of turbine & $F_{Y}$ & Lateral force of turbine \\
\hline$Z$ & Number of blades & $\lambda$ & Tip speed ratio \\
\hline$\omega$ & Angular velocity of turbine & $Q$ & Torque of turbine \\
\hline$\theta$ & Blade azimuth angle & $H$ & Blade length \\
\hline$\omega^{\prime}$ & Pitching angular velocity & $\xi$ & Pitching angle \\
\hline$A$ & Pitching amplitude & $R^{\prime}$ & Length of pitching arm \\
\hline$C$ & Chord length & $\omega_{z}$ & Pitching frequencies \\
\hline
\end{tabular}

The following dimensionless parameters are defined by

Tip speed ratio:

$$
\lambda=R \omega / V
$$

Thrust coefficient:

$$
C_{F X}=F_{X} /\left(0.5 \rho V^{2} D H\right)
$$

Lateral force coefficient:

$$
C_{F Y}=F_{Y} /\left(0.5 \rho V^{2} D H\right)
$$

Power coefficient:

$$
C_{P}=Q \omega /\left(0.5 \rho V^{3} D H\right)
$$

Dimensionless angular velocity of pitching motion:

$$
\bar{\omega}^{\prime}=\omega^{\prime} R^{\prime} / V
$$

Dimensionless angular acceleration of pitching motion:

$$
\vec{a}=\left(\partial_{t} \omega\right)^{\prime} R^{\prime 2} / V^{2}
$$

Pitching angle:

$$
\xi=A \sin \left(\omega_{z} t\right)
$$

According to the motion response of the floating structure and the symbolic characterization of physical quantities such as displacement, velocity and acceleration in waves, the coefficients of thrust and lateral forces can be divided into three components: the homogeneous hydrodynamic term, the damping term and the added mass term, respectively.

$$
\begin{gathered}
C_{F X}=C_{x}^{0}+n_{x x} \bar{\omega}^{\prime}+m_{x x} \bar{a}^{\prime} \\
C_{F Y}=C_{y}^{0}+n_{y y} \bar{\omega}^{\prime}+m_{y y} \bar{a}^{\prime} \\
\bar{\omega}^{\prime}=\frac{\omega^{\prime} R^{\prime}}{V}=\frac{\partial_{t} \xi R^{\prime}}{V}=\frac{A \omega_{z} \cos \left(\omega_{z} t\right) R^{\prime}}{V} \\
\vec{a}^{\prime}=\frac{\left(\partial_{t} \omega\right)^{\prime} R^{\prime 2}}{V^{2}}=\frac{\partial_{t}^{2} \xi R^{\prime 2}}{V^{2}}=\frac{-A \omega_{z}^{2} \sin \left(\omega_{z} t\right) R^{\prime 2}}{V^{2}}
\end{gathered}
$$

The damping and added mass terms are functions of $\theta$ (blade azimuth angle), which can be developed into triangular series functions as follows:

$$
\begin{aligned}
& n_{x x}=\frac{\delta C_{F X}}{\delta \bar{\omega}^{\prime}}=n_{x x}^{0}+\sum_{k=1}^{\infty} n_{x x}^{k} \sin \left(k z \theta(t)+\psi_{\omega^{\prime} x}^{k}\right) \\
& n_{y y}=\frac{\delta C_{F Y}}{\delta \bar{\omega}^{\prime}}=n_{y y}^{0}+\sum_{k=1}^{\infty} n_{y y}^{k} \sin \left(k z \theta(t)+\psi_{\omega^{\prime} y}^{k}\right) \\
& m_{x x}=\frac{\delta C_{F X}}{\delta \bar{a}^{\prime}}=m_{x x}^{0}+\sum_{k=1}^{\infty} m_{x x}^{k} \sin \left(k z \theta(t)+\psi_{a^{\prime} x}^{k}\right) \\
& m_{y y}=\frac{\delta C_{F Y}}{\delta \bar{a}^{\prime}}=m_{y y}^{0}+\sum_{k=1}^{\infty} m_{y y}^{k} \sin \left(k z \theta(t)+\psi_{a^{\prime} y}^{k}\right)
\end{aligned}
$$

Finally, the thrust and lateral force coefficients are expressed as follows:

$$
\begin{aligned}
& C_{F x}=C_{x}^{0}+n_{x} \vec{w}+m_{x} \vec{a}
\end{aligned}
$$

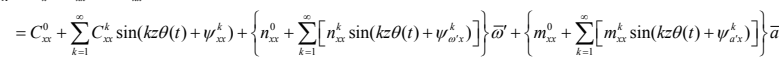

$$
\begin{aligned}
& C_{F Y}=C_{y}^{0}+n_{y y^{\prime}} \bar{\omega}^{\prime}+m_{y p^{\prime}} \vec{a}
\end{aligned}
$$

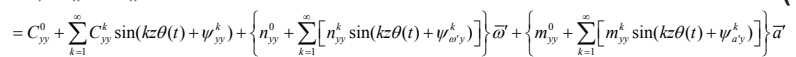

The time-varying curves for thrust and lateral force coefficients can be obtained by CFD. And then, by using the least squares method, the load coefficients for the homogeneous hydrodynamic, damping and added mass terms can also be obtained. ANSYS-CFX software can simulate small scale turbines and obtain relatively accurate calculation results. Because this paper mainly through the calculation of forced motion of the turbine load, and on this basis, combined with the theory of floating body in waves, the load of the turbine is decomposed and the hydrodynamic coefficients of different orders can be obtained, which can be 
coupled with the floating platform analysis. In such a case, the fittings according to different geometrical and environmental parameters, as well as the accuracy of the decomposition, have a great effect on the reliability of the obtained hydrodynamic coefficients. However, the least squares method is widely applied in curve fitting and is accurate enough. Therefore, the methodology adopted in this paper included the least squares method and CFD simulation for better robustness, and is applicable to turbines of different sizes.

\section{CFD SIMULATION}

The two-bladed vertical-axis turbine used in this simulation model was developed by Harbin Engineering University (HEU). Table 2 shows the detailed particulars of the HEU turbine model. A series of experiments was conducted in the circulating water tunnel laboratory of HEU. The two-bladed turbine is installed on a test platform, which is installed in the circulating water tunnel working segment $(8 \mathrm{~m} \times 1.7 \mathrm{~m} \times 1.5 \mathrm{~m})$. By changing the velocity, the hydrodynamic performance of the turbine can be studied under different work conditions, which can be tested for a long time (see. Fig. 1).

Tab. 2. Particulars of turbine model

\begin{tabular}{|l|c|c|c|}
\hline Item & Symbol & Data & Unit \\
\hline Diameter of turbine & $\mathrm{D}$ & 0.8 & $\mathrm{~m}$ \\
\hline Velocity & $\mathrm{V}$ & 1.0 & $\mathrm{~m} / \mathrm{s}$ \\
\hline Blade length & $\mathrm{H}$ & 0.6 & $\mathrm{~m}$ \\
\hline Chord & $\mathrm{C}$ & 0.12 & $\mathrm{~m}$ \\
\hline Blade airfoil & \multicolumn{3}{|c|}{ NACA0018 } \\
\hline Number of blades & $Z$ & 2 & \\
\hline
\end{tabular}

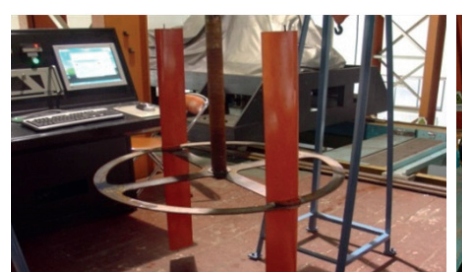

(a) Two-bladed vertical-axis tidal turbine

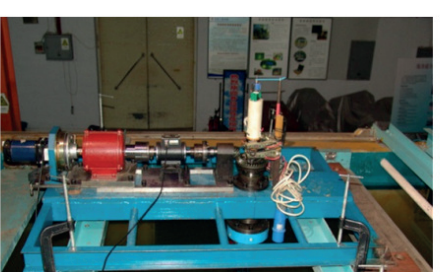

(b) Test platform

all CFD numerical simulations, the storage of structured grids can be recorded by a computer multidimensional array, and the simulation's precision and speed are significantly superior to those of unstructured grids. Therefore, structured grids are employed to divide the calculation domain of the turbine.

Fig. 2 shows the mesh grid models of the vertical-axis turbine. In order to fully simulate the vertical-axis turbine field characteristics, a sufficiently large computational domain needs to be established. The entire fluid domain employed in this paper is rectangular, with length $25 \mathrm{D}$ and width $40 \mathrm{D}$. The calculation domain is divided into three sections, namely rotation, pitching and static sections. They are connected by sliding mesh interfaces. In such a case, grid deformation does not exist during the simulation, which can ensure grid quality and improve the calculation precision. Quadrilateral structured $\mathrm{O}$ grids are used in three sections because of their high quality in dividing grids around cambered models. A pitching motion $\left(\xi=A \sin \left(\omega_{Z} t\right)\right)$ is applied to the rotation and pitching sections. Furthermore, rotary motion occurs concurrently around the centre of the rotation section, and the grids of the static section remain motionless.

The first layer of the turbine's surface meshes are defined as $0.00003 \mathrm{~m}$ especially, which ensures $\mathrm{Y}^{+}<2$. The total number of grids is 1200000 , and the SST model is chosen to simulate the vertical-axis turbine. The SST model proposed by Menter [11] in 1994 combines the advantages of $k-\varepsilon$ and $k-\omega$ models. It can also capture extreme pressures and velocity gradients in the vicinity of the turbine blades more accurately. It has been proven by many researchers, including Wang et al., Ponta and Dutt, and Shiono et al., that the SST model is more suitable for calculations of vertical-axis wind or water turbines $[13,15,18]$. Based on CFX software, the CEL code

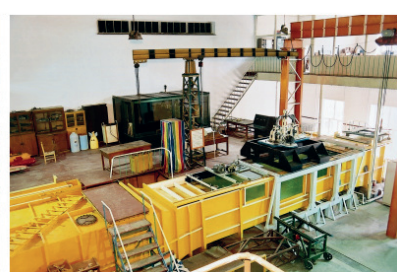

(c) Circulating water tunnel of HEU is programmed. According to previous research experience, the boundary conditions are set as follows. The reference atmospheric pressure is established, and the inlet boundary is set as entry speed with $V=1.0 \mathrm{~m} / \mathrm{s}$. The turbulence parameters and speeds are set simultaneously, and the outlet uses a pressure outlet boundary with a relative

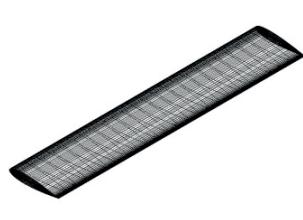

(a) Blade

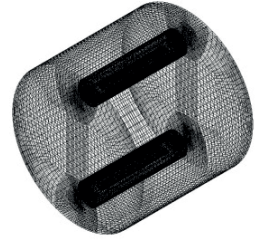

(b) Rotation section

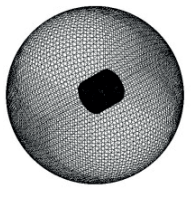

(c) Pitching section

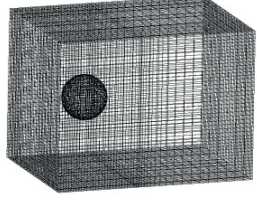

(d) Static section pressure of zero. The period during which the blade rotates $1^{\circ}$ is adopted as the time step of the entire simulation, and the blades rotate around the $\mathrm{Z}$ axis.

Fig. 2. Grid model

\section{CFD SIMULATION VERIFICATION}

Before using the CFD method to simulate the hydrodynamic performance of a turbine, the most significant work is to discretize the simulated area, namely by grid partitioning. For
The power coefficient of the turbine in constant inflow is illustrated in Fig. 3. The simulated and experimental values of power coefficients are in good accordance. The error between 
the results may come from the influence of free surface and wall on the hydrodynamic characteristics of the turbine, which are not considered in the calculation. This comparison result demonstrates that the CFD simulation method for tidal current turbines has satisfactory accuracy.

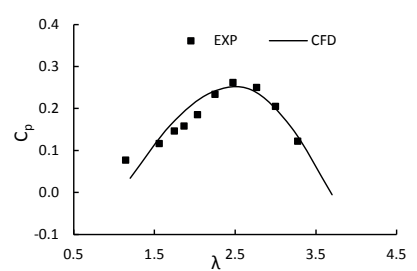

Fig. 3. Comparison of experimental and CFD simulation results

\section{SIMULATION RESULTS ANALYSIS}

The time-varying curves for thrust and lateral forces under non-pitching motion with an incoming flow velocity $V=1 \mathrm{~m} / \mathrm{s}$ and optimal turbine speed ratio $\lambda=2.5$ are shown in Fig. 4(a) and (b), respectively. Fig. 4(c) and (d) illustrate the time-varying curves for thrust and lateral forces under pitching motion $\left(A=10^{\circ}, W_{\mathrm{z}}=1.2 \mathrm{rad} / \mathrm{s}\right)$ with the same velocity and turbine speed ratio. In the second condition, the turbine revolves around the fixed main axis in uniform flow. The upper and lower envelopes of Fig. 4(a) and (b) are approximately straight lines.

However, the envelopes under pitching motion in Fig. 4(c) and (d) exhibit obvious cyclical fluctuations because of an added pitching motion. The main axis of the turbine undergoes a pitching movement when the turbine revolves around the main axis. Therefore, an additional fluctuation to that induced by turbine rotation itself is observed in the thrust and lateral forces of the turbine. In this paper, we study the hydrodynamic performance of vertical-axis tidal current turbines under pitching motion with different speed ratios, pitching frequencies and amplitudes.

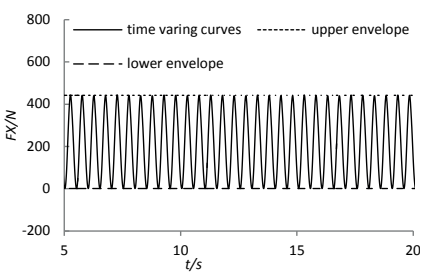

(a)

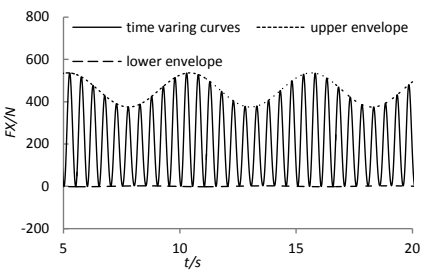

(c)

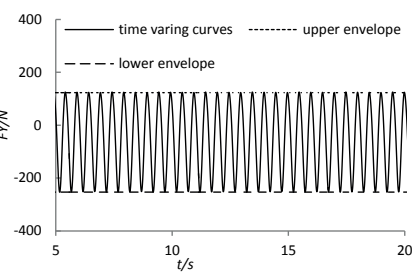

(b)

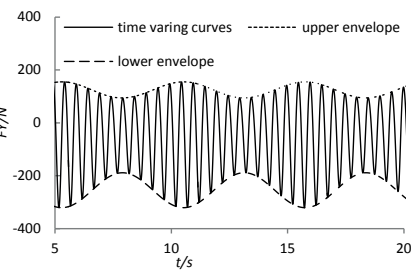

(d)
Fig. 4. Time-varying curves for thrust and lateral forces under pitching and non-pitching motion

\section{TIP SPEED RATIOS}

The power coefficient is the most significant factor for the initial design of a turbine. Therefore, the power coefficients of turbines were first compared under pitching and nonpitching motion. Fig. 5(a) presents the differences in power coefficients for the two motions with different tip speed ratios. The power coefficients in the former motion are obviously higher than those in the latter one, especially at middle and high tip speed ratios. Compared with turbines under nonpitching motion, the fluid speed distribution around the turbine of pitching motion changed because the additional pitching motion changes the transient force and torque of the turbine. Therefore, the transient values of the power coefficient under pitching motion will be higher. Then we compared the blade tangential force between non-pitching and pitching motion. The transient values of blade tangential force reflect the power coefficient of turbines directly.

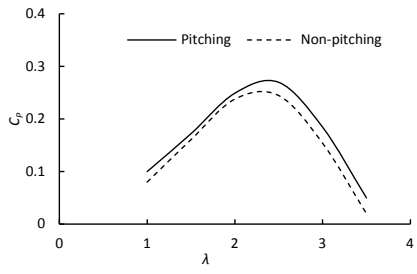

(a)

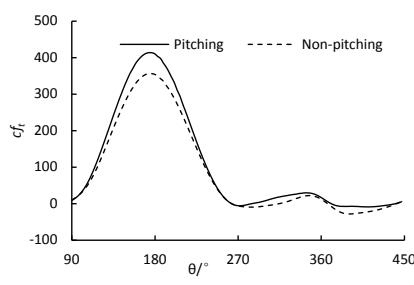

(b)
Fig. 5. Comparison of power coefficients and tangential force between nonpitching and pitching motion

As shown in Fig. 5(b), the power coefficient $c f_{t}$ of pitching motion is obviously higher than that of non-pitching motion at a blade azimuth angle approximately from $120^{\circ}$ to $200^{\circ}$. The upstream disk is the main work area for the turbine because the downstream values are almost zero. In consequence, the power coefficient under pitching motion is higher than that under non-pitching motion.

The thrust and lateral force envelope curves under pitching motion with five different tip speed ratios $(\lambda=1.5,2.0,2.5$, $3.0,3.5)$ are also considered in this study. The envelope curves for thrust and lateral forces on the turbine should be stable if the pitching motion is not taken into consideration. The pitching frequency is set as $1.2 \mathrm{rad} / \mathrm{s}$, and the amplitude is $10^{\circ}$. Fig. 6 shows the upper and lower envelope thrust curves in panels (a) and (b) and the lateral force in panels (c) and (d). The envelope curves for thrust and lateral forces under pitching motion fluctuate obviously. As the tip speed ratio increases, the upper envelope curves for thrust and lateral forces increase obviously. On the contrary, the lower envelope curves decrease with a rising tip speed ratio. The oscillation of thrust and lateral force of turbines under pitching motion impedes the stability of the output electricity and the security of the structure. 


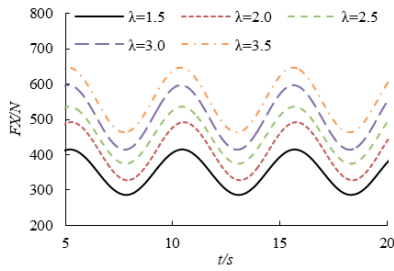

(a)

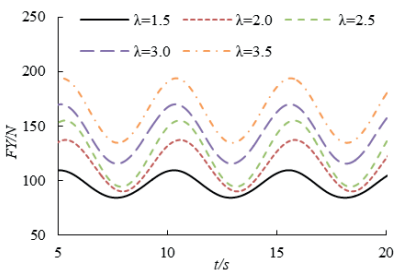

(c)

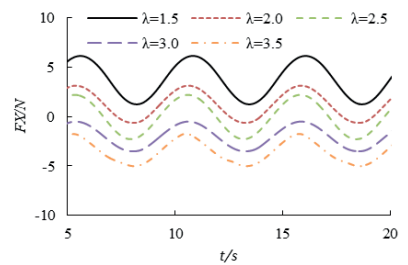

(b)

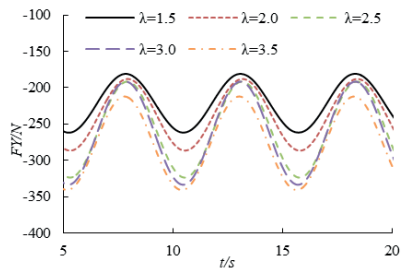

(d)
Fig. 6. Envelope curves for thrust and lateral forces of pitching motion under different tip speed ratios

The fluctuation amplitudes are defined by $\widehat{F X}=F X_{\max }-F X_{\min }$ and $\widehat{F Y}=F Y_{\max }-F Y_{\min }$. Fig. 7 shows the fluctuation amplitudes of thrust and lateral forces with different tip speed ratios. It is shown that the fluctuation amplitudes increase gradually with increasing tip speed ratios. The fluctuation amplitude under pitching motion is obviously higher than that under non-pitching motion with the same tip speed ratio. When the tip speed ratio is 2.5 , the thrust fluctuation amplitude under pitching motion can be up to $29 \%$ larger than that under non-pitching motion, with a $28 \%$ increase for lateral force. Therefore, when the turbine works in waves and current, the structure should be strengthened because there will be pitching motion.

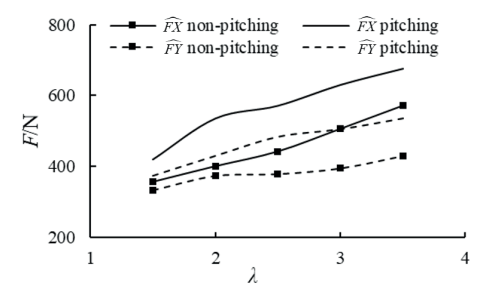

Fig. 7. Comparison of fluctuation amplitude under different tip speed ratios

\section{PITCHING FREQUENCIES}

The pitching frequencies of the turbine are related to wave frequencies. Therefore, the pitching frequencies were selected as $0.4,0.8,1.2,1.6$, and $2.0 \mathrm{rad} / \mathrm{s}$, respectively. The tip speed ratio of the turbine was set as 2.5 and the pitching amplitude is $10^{\circ}$.

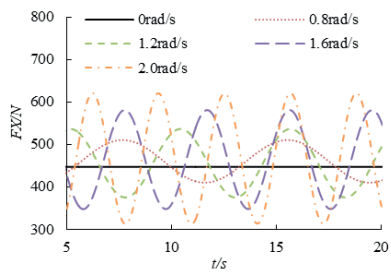

(a)

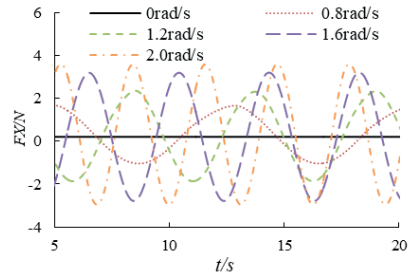

(b)

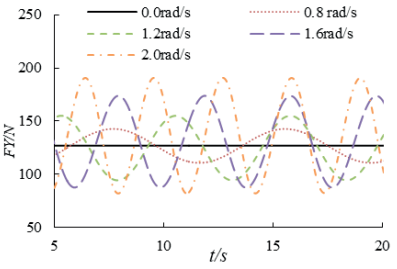

(c)

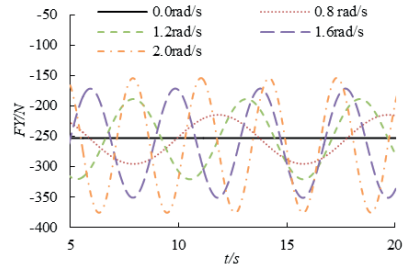

(d)
Fig. 8. Envelope curves for thrust and lateral forces of pitching motion with different frequencies

Fig. 8 shows how the envelope curves for thrust and lateral forces under different pitching frequencies change with time. In the following figures, the graphic legends represent the pitching frequencies and $0 \mathrm{rad} / \mathrm{s}$ means non-pitching. The upper and lower envelope curves for thrust are shown in subgraphs (a) and (b) and those for lateral force in subgraphs (c) and (d), respectively.

When the turbine works in waves, the load envelope curves fluctuate, with the envelope curves for a non-pitching load as the baseline. The upper envelope curves' fluctuation amplitude of thrust is obviously higher than that of the lower envelope curves, and the lateral force of the envelope curves fluctuates with almost same the order of magnitude. According to the graphs, as the pitch frequency increases, their fluctuation amplitudes rise while their periods decrease. For example, when the pitching frequency is 2.0 , the fluctuation amplitude of the lateral force's upper envelope curve can be as large as $85.1 \%$ of the non-pitching value and $87.6 \%$ for the lower envelope curve. The pitching motion changes the fluid speed distribution around the turbine, which links to the transient force and torque of the turbine. Therefore, the loads on the turbine oscillate with high frequency and large amplitude when high-frequency waves occur, and the turbine structure should be strengthened in high-frequency waves.

The mean values and fluctuation amplitudes are defined by

$\overline{F X}=\int_{t_{1}}^{t_{2}} F X d t /\left(t_{2}-t_{1}\right), \widehat{F X}=F X_{\max }-F X_{\min }, \overline{F Y}=\int_{t_{1}}^{t_{2}} F Y d t /\left(t_{2}-t_{1}\right)$

and $\widehat{F Y}=F Y_{\max }-F Y_{\min }$. The mean values and fluctuation amplitudes of thrust and lateral forces under the designed working conditions (incoming speed $1.0 \mathrm{~m} / \mathrm{s}$, tip speed ratio 2.5 and amplitude $10^{\circ}$ ) are shown in Table 3 . We can conclude that the mean values have minimal influence with increasing pitching frequencies and that the fluctuation amplitudes increase gradually. For example, when the pitching frequency is $2.0 \mathrm{rad} / \mathrm{s}$, the fluctuation amplitude of thrust force can be up to $41.3 \%$ higher than that in non-pitching motion, with a $71.3 \%$ increase for lateral force.

Tab. 3. Comparison of average values and fluctuation amplitudes

\begin{tabular}{|c|c|c|c|c|}
\hline$\omega_{z}$ & $\overline{F X}$ & $\widehat{F X}$ & $\overline{F Y}$ & $\widehat{F Y}$ \\
\hline 0 & 218.37 & 442.19 & -51.90 & 378.43 \\
\hline 0.4 & 221.69 & 510.97 & -48.57 & 400.87 \\
\hline 0.8 & 220.64 & 522.97 & -49.78 & 447.93 \\
\hline 1.2 & 218.35 & 572.20 & -51.03 & 508.98 \\
\hline 1.6 & 222.82 & 614.55 & -51.36 & 585.89 \\
\hline 2.0 & 223.08 & 624.81 & -51.04 & 648.46 \\
\hline
\end{tabular}




\section{PITCHING AMPLITUDES}

The calculation results with a tip speed ratio of 2.5 and pitching frequency of $1.2 \mathrm{rad} / \mathrm{s}$ considering different pitching amplitudes $\left(0^{\circ}, 5^{\circ}, 10^{\circ}\right.$ and $\left.15^{\circ}\right)$ are shown in Fig. 9. Here, $0^{\circ}$ means no pitching motion occurs. Similarly, the envelope curves for hydrodynamic loads fluctuate with the baseline defined by the envelope curves for non-pitching loads, when the turbine pitches in waves. When the pitching amplitude increases, the amplitudes of envelope curves increase, while their mean values remain stable. Increasing pitching amplitude has a negative impact on structural security. It also adds difficulty to the control of electricity output because of the fluctuation induced by the pitching motion.

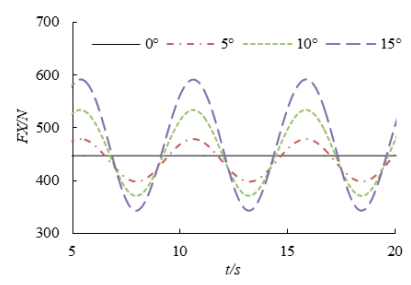

(a)

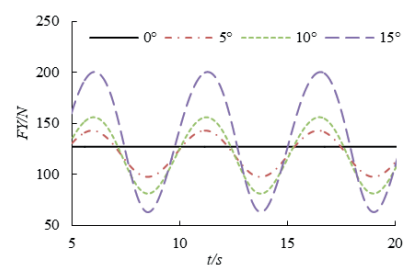

(c)

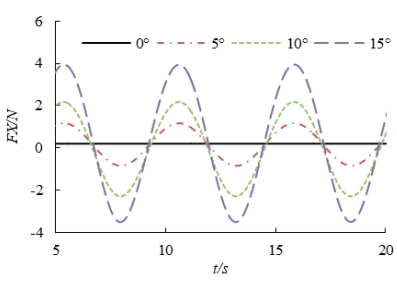

(b)

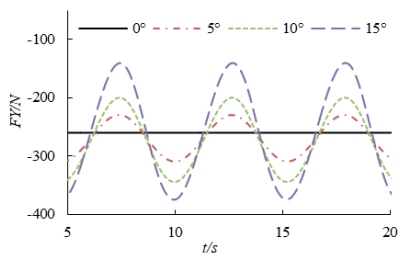

(d)
Fig. 9. Envelope curves for thrust and lateral force for pitching motion under different amplitudes

\section{FITTING RESULTS ANALYSIS}

Time-varying curves with different tip speed ratios, pitching frequencies and amplitudes were obtained by CFD simulation. Based on the former simulation results, using the least squares method, the added mass and damping coefficients can be obtained. The fitting and CFD simulation results for a given condition $\left(V_{A}=1.0 \mathrm{~m} / \mathrm{s}, \lambda=2.5, \omega_{Z}=1.2 \mathrm{rad} / \mathrm{s}, \mathrm{A}=10^{\circ}\right)$ are shown in Fig. 10. Obviously, the CFD simulation results can be perfectly fitted by using the least squares method.

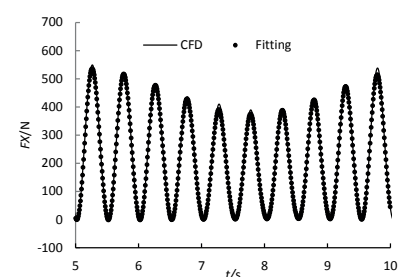

(a)

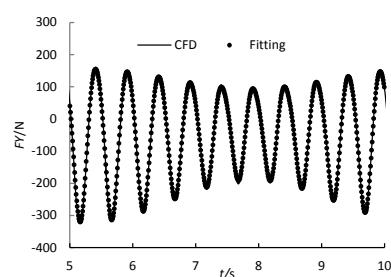

(b)
Fig. 10 Comparison between fitting and CFD results

\section{TIP SPEED RATIO}

Coefficients of $C_{F X}$ and $C_{F Y}$ series were obtained by fitting the time-varying curves for thrust and lateral forces with different tip speed ratios. Table 4 shows the coefficients for the constant terms and first-order terms of $C_{F X}$ and $C_{F Y^{*}}$

Tab. 4(a). $C_{F X}$ expansion coefficients under different tip speed ratios

\begin{tabular}{|c|c|c|c|c|c|c|c|c|c|}
\hline$\lambda$ & $C_{x x}^{0}$ & $C_{x x}^{1}$ & $\psi_{x x}^{1}$ & $n_{x x}^{0}$ & $n_{x x}^{1}$ & $\psi_{\omega^{\prime} x}^{1}$ & $m_{x x}^{0}$ & $m_{x x}^{1}$ & $\psi_{a^{\prime} x}^{1}$ \\
\hline 1.5 & 0.688 & 0.711 & -80.235 & 0.905 & 0.930 & -78.198 & -0.011 & 0.074 & -130.239 \\
\hline 2.0 & 0.839 & 0.858 & -80.783 & 1.296 & 1.357 & -86.297 & -0.017 & 0.090 & 120.862 \\
\hline 2.5 & 0.915 & 0.946 & -82.431 & 1.485 & 1.366 & -76.936 & -0.024 & 0.122 & -122.325 \\
\hline 3.0 & 0.980 & 1.050 & -85.127 & 1.679 & 1.481 & -83.093 & -0.064 & 0.150 & -75.114 \\
\hline 3.5 & 1.033 & 1.157 & -86.128 & 1.696 & 1.520 & -89.522 & -0.073 & 0.178 & -60.948 \\
\hline
\end{tabular}

Tab. 4(b). $C_{F Y}$ expansion coefficients under different tip speed ratios

\begin{tabular}{|c|c|c|c|c|c|c|c|c|c|}
\hline$\lambda$ & $C_{y y}^{0}$ & $C_{y y}^{1}$ & $\psi_{y y}^{1}$ & $n_{y y}^{0}$ & $n_{y y}^{1}$ & $\psi_{\omega^{\prime} y}^{1}$ & $m_{y y}^{0}$ & $m_{y y}^{1}$ & $\psi_{a^{\prime} y}^{1}$ \\
\hline 1.5 & -0.196 & 0.69 & 177.91 & -0.44 & 1.57 & -150.11 & 0.01 & 0.16 & 102.94 \\
\hline 2.0 & -0.198 & 0.75 & 171.67 & -0.45 & 1.61 & 164.48 & -0.01 & 0.19 & -27.09 \\
\hline 2.5 & -0.204 & 0.79 & 173.98 & -0.47 & 1.62 & 169.51 & -0.02 & 0.20 & -35.23 \\
\hline 3.0 & -0.207 & 0.81 & 175.37 & -0.49 & 1.65 & 164.07 & -0.04 & 0.24 & -121.85 \\
\hline 3.5 & -0.209 & 0.83 & 178.62 & -0.51 & 1.67 & 166.57 & -0.07 & 0.25 & -151.98 \\
\hline
\end{tabular}

It is concluded from Table 4(a) that with an increase of tip speed ratio, the absolute values of constant terms and first-order terms for homogeneous hydrodynamic, damping and added mass coefficients increase obviously. However, compared with the values for damping coefficients, added mass coefficients are significantly lower. Table 4(b) shows similar changing trends for the constant terms and firstorder terms for the coefficients. Therefore, the time-varying oscillations of $C_{F X}$ and $C_{F Y}$ induced by pitching motion are mainly attributed to the damping terms, and their fluctuation amplitude increases with an increase in tip speed ratio.

\section{PITCHING FREQUENCIES}

Series coefficients of $C_{F X}$ and $C_{F Y}$ are obtained by fitting the time-varying curves for thrust and lateral forces with different pitching frequencies; their constant and first-order terms are shown in Table 5.

Tab. 5(a). $C_{F X}$ expansion coefficients under different frequencies

\begin{tabular}{|r|c|c|c|c|c|c|c|c|r|}
\hline$\omega_{Z}$ & $C_{x x}^{0}$ & $C_{x x}^{1}$ & $\psi_{x x}^{1}$ & $n_{x x}^{0}$ & $n_{x x}^{1}$ & $\psi_{\omega^{\prime} x}^{1}$ & $m_{x x}^{0}$ & $m_{x x}^{1}$ & $\psi_{a^{\prime} x}^{1}$ \\
\hline 0.40 & 0.914 & 0.951 & -83.544 & 1.348 & 1.207 & -83.125 & -0.039 & 0.125 & 91.337 \\
\hline 0.80 & 0.913 & 0.954 & -83.027 & 1.356 & 1.272 & -74.697 & -0.090 & 0.152 & -117.685 \\
\hline 1.20 & 0.915 & 0.946 & -82.431 & 1.485 & 1.366 & -76.936 & -0.024 & 0.122 & -122.325 \\
\hline 1.60 & 0.913 & 0.946 & -82.296 & 1.530 & 1.460 & -76.942 & -0.063 & 0.223 & -102.953 \\
\hline 2.00 & 0.911 & 0.952 & -82.163 & 1.543 & 1.539 & -76.812 & -0.010 & 0.122 & -98.097 \\
\hline
\end{tabular}

Tab. 5(b). $C_{F Y}$ expansion coefficients under different frequencies

\begin{tabular}{|c|c|c|c|c|c|c|c|c|r|}
\hline$\omega_{Z}$ & $C_{y y}^{0}$ & $C_{y y}^{1}$ & $\psi_{y y}^{1}$ & $n_{y y}^{0}$ & $n_{y y}^{1}$ & $\psi_{\omega^{\prime} y}^{1}$ & $m_{y y}^{0}$ & $m_{y y}^{1}$ & $\psi_{a^{\prime} y}^{1}$ \\
\hline 0.40 & -0.203 & 0.79 & 174.29 & -0.57 & 1.93 & -175.39 & -0.07 & 0.23 & 23.75 \\
\hline 0.80 & -0.209 & 0.79 & 173.77 & -0.51 & 1.72 & 170.65 & -0.06 & 0.11 & -102.94 \\
\hline 1.20 & -0.204 & 0.79 & 173.98 & -0.47 & 1.62 & 169.51 & -0.02 & 0.20 & -35.23 \\
\hline 1.60 & -0.204 & 0.81 & 174.01 & -0.43 & 1.58 & 168.36 & -0.02 & 0.19 & -58.02 \\
\hline 2.00 & -0.202 & 0.80 & 173.72 & -0.40 & 1.56 & 168.10 & -0.06 & 0.23 & -54.01 \\
\hline
\end{tabular}


Table 5(a) and (b) show that the fitting homogeneous hydrodynamic expansion coefficients $\left(C_{x x}^{0}, C_{x x}^{1}, C_{y y}^{0}\right.$ and $C_{y y}^{1}$ ) of the turbine's thrust and lateral forces are not related to the acceleration and velocity of the pitching motion. The constant and first-order terms of the homogeneous hydrodynamic coefficient will remain stable with the growth of pitching frequencies. When the frequencies increase, the constant and first-order terms of damping coefficients in Table 5(a) will increase obviously and the corresponding terms (absolute values) of damping coefficients in Table 5(b) oppositely decrease. The tables also show that the added mass coefficient is one order of magnitude lower than the homogeneous hydrodynamic and damping coefficients. Therefore, the main factors that induce the time-varying fluctuation of $C_{F X}$ and $C_{F Y}$ under pitching motion are the damping terms.

\section{PITCHING AMPLITUDES}

Similarly, by fitting the time-varying curves for the thrust and lateral forces with different pitching amplitudes, the series coefficients of $C_{F X}$ and $C_{F Y}$ are obtained. Their constant and first-order terms are shown in Table 6.

Tab. 6(a). CFX expansion coefficients under different amplitudes

\begin{tabular}{|c|c|c|c|c|c|c|c|c|c|}
\hline$A$ & $C_{x x}^{0}$ & $C_{x x}^{1}$ & $\psi_{x x}^{1}$ & $n_{x x}^{0}$ & $n_{x x}^{1}$ & $\psi_{\omega^{\prime} x}^{1}$ & $m_{x x}^{0}$ & $m_{x x}^{1}$ & $\psi_{a^{\prime} x}^{1}$ \\
\hline 5 & 0.917 & 0.949 & -82.628 & 1.478 & 1.347 & -78.487 & -0.028 & 0.121 & 123.312 \\
\hline 10 & 0.915 & 0.946 & -82.431 & 1.485 & 1.366 & -76.936 & -0.024 & 0.122 & -122.325 \\
\hline 15 & 0.919 & 0.945 & -82.052 & 1.498 & 1.403 & -76.768 & -0.029 & 0.125 & 122.225 \\
\hline
\end{tabular}

Tab. 6(b). CFY expansion coefficients under different amplitudes

\begin{tabular}{|c|c|c|c|c|c|c|c|c|c|}
\hline$A$ & $C_{y y}^{0}$ & $C_{y y}^{1}$ & $\psi_{y y}^{1}$ & $n_{y y}^{0}$ & $n_{y y}^{1}$ & $\psi_{\omega^{\prime} y}^{1}$ & $m_{y y}^{0}$ & $m_{y y}^{1}$ & $\psi_{a^{\prime} y}^{1}$ \\
\hline 5 & -0.205 & 0.788 & 172.691 & -0.480 & 1.632 & 169.267 & 0.01 & 0.21 & 45.761 \\
\hline 10 & -0.204 & 0.791 & 173.98 & -0.470 & 1.621 & 169.51 & -0.02 & 0.20 & -35.23 \\
\hline 15 & -0.201 & 0.801 & 174.838 & -0.434 & 1.617 & 166.230 & -0.01 & 0.23 & 42.092 \\
\hline
\end{tabular}

As seen from Table 6, the main conclusions for different amplitudes are similar to the conclusions given in the section 'CFD SIMULATION'. As the pitching amplitude increases, the constant and first-order terms of the homogeneous hydrodynamic coefficients remain stable. The absolute values of constant and first-order terms for damping coefficients in Table 6(a) increase with the growth of amplitudes, but the absolute values of the corresponding terms for damping coefficients in Table 6(b) decrease significantly. What is more, the constant and first-order terms for added mass in the two tables are lower by an order of magnitude. Therefore, the fluctuation induced by pitching motion under different amplitudes is mainly concerned with the damping coefficient.

\section{CONCLUSIONS}

Floating vertical-axis tidal current turbines undergo motion in six degrees of freedom induced by waves and currents. Pitching motion only belongs to one of the six degrees of freedom. The methodology of this paper including the least squares method and CFD simulation can also be used to calculate the hydrodynamic performance for other freedoms of motion, such as sway and surge. This will be the key points of our study in the future. The main content of this paper is to calculate the added mass and damping coefficients for a turbine caused by waves. In such a case, by introducing these results, it is possible to conduct coupled dynamic analysis between the floating platform, mooring system and tidal current turbines in time and frequency domains.

The pitching motions of vertical-axis tidal current turbines under different tip speed ratios, frequencies and amplitudes were simulated by CFX software, and then the least squares fitting method was used to fit time-varying curves and obtain added mass and damping coefficients. The study demonstrates that:

(1) The fluctuation of a vertical-axis turbine induced by waves and currents cannot be neglected during the design stage, because it can cause the fluctuation of thrust and lateral forces. The fluctuation amplitudes of thrust and lateral forces have a positive correlation with pitching tip speed ratios, frequencies and amplitudes. At a high pitching frequency such as $2.0 \mathrm{rad} / \mathrm{s}$, the fluctuation amplitude of the thrust force can be up to $41.3 \%$ higher than that under non-pitching motion, with a $71.3 \%$ increase for lateral force. Therefore, the fluctuation induced by waves and currents will have obvious negative impacts on the turbine's structural security, and turbines under pitching motion require a greater structural strength to extend the turbine's fatigue life.

(2) Compared with turbines under non-pitching motion, the pitching motion induced by waves and currents has a positive effect on the power output of turbines, which will increase the tangential force of blades during the upstream disk.

(3) The mean values of thrust and lateral forces are marginally affected by pitching frequencies and pitching amplitudes. However, increasing tip speed ratio has a positive effect on the average (absolute values) thrust and lateral forces.

(4) The thrust and lateral forces of the turbine are analysed with the least squares method. The damping coefficient plays a more important role than added mass in the fluctuation of loads induced by pitching motion. The constant and firstorder terms of damping coefficients have a positive correlation with tip speed ratios, frequencies and amplitudes. These can provide some useful references for the motion response analysis (in time or frequency domain) of floating tidal current power stations. 


\section{ACKNOWLEDGEMENTS}

This paper is financially supported by the National Natural Science Foundation of China (No. 51909111) and the Natural Science Foundation of Jiangsu Province (No. BK20180980).

\section{REFERENCES}

1. Chowdhury A. M., Akimoto H., Hara Y. (2016): Comparative CFD analysis of vertical axis wind turbine in upright and tilted configuration. Renewable Energy, Vol. 85(1), 327-337.

2. Dai J., Shan Z. D., Wang X. F. (2010): Current research progress of water turbine. Renewable Energy, Vol. 28(4), $130-133$

3. Galloway P. W., Myers L. E., Bahaj A. S. (2010): Studies of a scale tidal turbine in close proximity to waves. In: Third International Conference and Exhibition on Ocean Energy, Bilbao, Spain, 6-8 Oct, 2010.

4. Li B. Y., Karri N., Wang Q. (2014): Three-dimensional numerical analysis on blade response of a vertical-axis tidal current turbine under operational conditions. Journal of Renewable and Sustainable Energy, Vol. 6(4), 043123.

5. Li G. N., Chen Q. R., Gu H. B. (2018): An unsteady boundary element model for hydrodynamic performance of a multiblade vertical-axis tidal turbine. Water, Vol. 10, 1413.

6. Li G. N., Chen Q. R., Gu H. B. (2018): Study of hydrodynamic interference of vertical-axis tidal turbine array. Water, Vol. $10,1228$.

7. Li G. N., Chen Q. R., et al. (2020): Study on hydrodynamic configuration parameters of vertical-axis tidal turbine. Polish Maritime Research, Vol. 27(1): 8-15.

8. Li Y., Calisal S. M. (2011): Modeling of twin-turbine systems with vertical axis tidal current turbines: Part I-Power output. Ocean Engineering, Vol. 37(7), 627-637.

9. Li Y., Calisal S. M. (2011): Modeling of twin- turbine systems with vertical axis tidal current turbines: Part II-Torque fluctuation. Ocean Engineering, Vol. 38(4), 550-558.

10. Li Z. C. (2011): Numerical Simulation and Experimental Study on Hydrodynamic Characteristic of Vertical Axis Tidal Turbine. Doctoral thesis, Harbin Engineering University, (in Chinese).

11. Menter F. R. (1994): Two-equation eddy-viscosity turbulence models for engineering applications. AIAA Journal, Vol. 32(8), 1598-1605.
12. Ponta F., Dutt G. S. (2000): An improved vertical axis water current turbine incorporating a channeling device. Renewable Energy, Vol. 20(2), 223-241.

13. Ponta F. L., Jacovkis P. M. (2011): A vortex model for Darrieus turbine using finite element techniques. Renewable Energy, Vol. 24(1), 1-18.

14. Scheurich, F., Brown, R. E. (2011): Vertical-axis wind turbines in oblique flow: sensitivity to rotor geometry. In: European Wind Energy Conference and Exhibition Brussels, Belgium, 14-17 Mar, 2011.

15. Shiono M., Suzuki K., Kiho S. (2002): Output characteristics of Darrieus water turbine with helical blades for tidal current generations. In: Proceedings of 12th International Offshore and Polar Engineering Conference, Kitakyushu, Japan, 26-31 May, 2002.

16. Tomporowski A., Al-Zubiedy A., et al. (2019): Analysis of the project of innovative floating turbine. Polish Maritime Research, Vol. 26(4): 124-133.

17. Wang S., Yuan P., Li D., Jiao Y. (2011): An overview of ocean renewable energy in China. Renewable and Sustainable Energy Reviews, Vol. 15(1), 91-111.

18. Wang S. Y., Derek B. I., Ma L., Pourkashanian M., Tao Z. (2012): Turbulence modeling of deep dynamic stall at relatively low Reynolds number. Journal of Fluids and Structures, Vol. 33(8), 191-209.

19. Yang B., Lawn C. (2011): Fluid dynamic performance of a vertical axis turbine for tidal currents. Renewable Energy, Vol. 36(12), 3355-3366.

20. Zhang Z. Y., Ma Y., et al. (2017): 3-D simulation of verticalaxial tidal current turbine. Polish Maritime Research, Vol. 23(4): 73-83.

\section{DATA AVAILABILITY STATEMENT}

The CFD simulation raw data used to support the findings of this study are available from the corresponding author upon request. 


\section{CONTACT WITH THE AUTHORS}

\section{Wanchao Zhang}

e-mail: zhangwanchao@just.edu.cn

Jiangsu University of Science and Technology,

No.2 Mengxi Road, 212000 Zhenjaing,

\section{China}

\section{Yujie Zhou}

e-mail:zhouyujie@just.edu.cn

Jiangsu University of Science and Technology, Mengxi Road, 212000 Zhenjiang

\section{China}

\section{Kai Wang}

e-mail:wangkai198949@qq.com

Wuhan Second Ship Design \& Research Institute, 450 Zhongshan Road, 430064 Wuhan, China

China

\section{Xiaoguo Zhou}

e-mail:zhouxiaoguo@just.edu.cn

Jiangsu University of Science and Technology, Mengxi Road, 212000 Zhenjiang, China

\section{CHINA}

